\title{
Decision Support: Time for Collaboration
}

\author{
A. Guardia, Section Editor for the IMIA Yearbook Section on Decision Support \\ Department of Medical Imaging and Information Sciences, Division of eHealth and Telemedicine \\ Geneva University Hospitals, Geneva, Switzerland
}

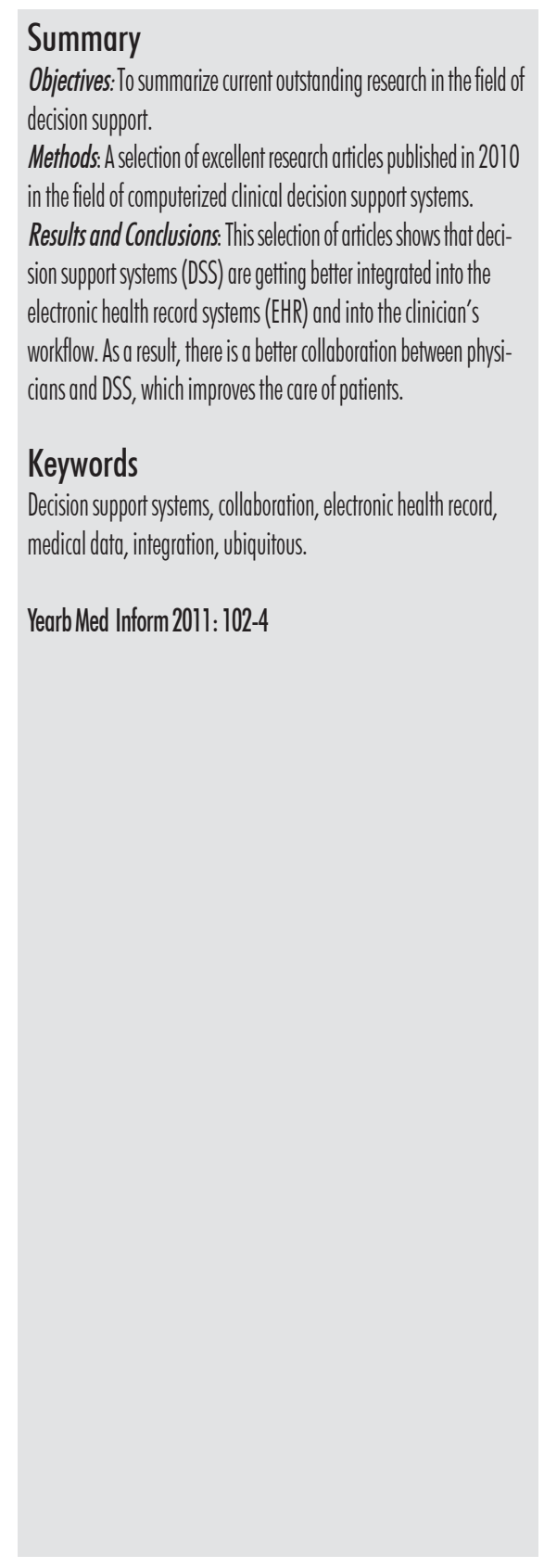

\section{Introduction}

During the last decade, the domain of medical decision support systems (DSS) has grown thanks to an improved integration with electronic health records (EHRs) [10]. DSS can thus more actively collaborate with physicians in order to provide advice on diagnosis, drug interactions or on imaging $[1,2,3,5,12]$.

Recent publications on decision support show that DSS are also better integrated into the workflow of the physician (i.e. information is provided when useful for the physician and is easily accessible for him).

DSS are also shown to improve the quality of inpatient care, reduce the physician's learning curve as well as the cost of inpatient stays [1,13].

Moreover, the ability to combine multiple DSS increases the relevance of their answers [2].

Finally, we clearly see that the access and the use of qualitative data are as important as the integration in the physician workflow. In this context, future DSS technology will probably be enhanced by mobile tools, which will lead to ubiquitous access to information and decision support tools $[7,11]$.

\section{Best Paper Selection}

For the section Decision Support on the Health Information System in the IMIA Yearbook 2011 (Table 1), we selected five papers demonstrating the possibilities and advantages of a mature DSS integrated to an EHR. A brief summary of these articles is provided in the appendix section.

\section{Conclusions and Outlook}

As mentioned in our selection of articles for Decision Support section in the Yearbook 2011, the decision support is currently sufficiently developed to work properly with data from medications, and clinical and laboratory results. This collaboration allows to identify probable gaps in the problem list $[3,8,9]$ and to prevent possible diseases [4]. In both cases, the inpatient is the beneficiary.

Obviously, this level of performance has several impacts on the patient, the physician and the hospitals. Indeed, as demonstrated in Elkin's paper, if the tool of the decision support correctly utilizes the clinical information's data, it may improve the patient's care, refine the training of doctors and reduce hospital stay and therefore costs $[1,13]$.

As a conclusion, it appears through this selection of articles that decision support is entering a new stage, where its performance allows to act as an adviser for doctors and where its effectiveness is a reflection of an increasingly powerful and innovative technology with the use of optimized clinical information data.

\section{Acknowledgement}

I greatly acknowledge the support of Martina Hutter and of the reviewers in the selection process of the IMIA Yearbook.

\section{References}

1. Elkin PL, Liebow M, Bauer BA, Chaliki S, Wahner-Roedler D, Bundrick J, et al. The introduction of a diagnostic decision support system (DXplain ${ }^{\mathrm{TM}}$ )into the workflow of a teaching hospital service can decrease the cost of service for diagnostically challenging Diagnostic Related Groups (DRGs). Int J Med Inform 2010;79:772-7.

2. Niemeijer M, Loog M, Abramoff MD, Viergever 
MA, Prokop M, van Ginneken B. On combining Computer-Aided Detection Systems. IEEE Trans Med Imaging 2010;30:215-23.

3. Wright A, Chen ES, Maloney FL. An automated technique for identifying associations between medications, laboratory results and problems. J Biomed Inform 2010; 43:891-901.

4. Matheny ME, Miller RA, IkizlerTA, Waitman LR, Denny JC, Schildcrout JS, et al. Development of inpatient risk stratification models of acute kidney injury for use in electronic health record. Med Decis Making 2010;30:639-50.

5. van der Sijs H, van Gelder T, Vulto A, Berg M, Aarts J. Understanding handling of drug safety alerts: a simulation study. Int J Med Inform 2010;79:361-9.

6. Ruch P, A Medical Informatics Perspective on Decision Support. Toward a Unified Research Paradigm Combining Biological vs. Clinical, Empirical vs. Legacy,and Structured vs. Unstructured Data. Yearb Med Inform 2009;96-8.

7. Ahmadian L, van Engen-Verheul M, Bakhshi-Raiez F, Peek N, Cornet R, de Keizer NF. The role of standardized data and terminological systems in computerized clinical decision support systems: Literature review and survey. Int J Med Inform 2010; 80(2):81-93

8. Galanter WL, Hier DB, Jao C, Sarne D. Computerized physician order entry of medications and clinical decisions support can improve problem list documentation compliance. Int J Med Inform 2010;79:332-8.

9. Goud R, van Engen-Verheul M, de Keizer NF, Bal R, Hasman A, Hellemans IM, et al. The effect of computerized decision support on barriers to guideline implementation: A qualitative study in outpatient cardiac rehabilitation. Int $\mathrm{J}$ Med Inform 2010;79:430-7.

10. Shim JP, Warkentin M, Courtney JF, Power DJ, Sharda R, Carlsson C. Past,present and future of decision support technology. Decis Support Syst 2002;33:111-26.

11. Eren A, Subasi A, Coskun O. A Decision Support System for Telemedicine Through the Mobile Telecommunications Platform. J Med Syst 2008;32(1):31-5.

12. Kawamoto K, Houlihan CA, Balas EA, Lobach DF. Improving clinical practice using clinical decision support systems: a systematic review of trials to identify features critical to success. BMJ 2005;330(7494):765.

13. Garg AX, Adhikari NKJ, McDonald H, RosasArellano MP, Devereaux PJ, Beyene J, et al. Effects of Computerized Clinical Decision Support Systems on Practitioner Performance and Patient Outcome. JAMA 2005;293(10):1223-38.

Correspondence to:

Alberto Guardia, MD.

University Hospitals of Geneva

Department of medical imaging and information sciences

Division of eHealth and telemedicine

4, Rue Gabrielle-Perret-Gentil

CH-1211 Geneva 14, Switzerland

Tel: +41 223726293

Fox: +41 223728680

Email: alberto.guardia@hcuge.ch

Table 1 Best paper selection of articles for the IMIA Yearbook of Medical Informatics 2011 in the section 'Decisin Support'. The articles are listed in alphabetical order of the first author's surname.

Section

Decision Support

- Elkin PL, Liebow M, Bauer BA, Chaliki S, Wahner-Roedler D, BundrickJ, Lee M, Brown SH, Froehling D, Bailey K, Famiglietti K, Kim R, Hoffer E, Feldman M, Barnett GO. The introduction of a diagnostic decision support system (DXplain ${ }^{T M}$ ) into the workflow of a teaching hospital service can decrease the cost of service for diagnostically challenging Diagnostic Related Groups (DRGS). IntJ Med Inform 2010;79:772-7.

- Matheny ME, Miller RA, Ikizler TA, Waitman LR, Denny JC, Schildcrout JS, Dittus RS, Peterson JF. Development of inpatient risk stratification models of acute kidney injury for use in electronic health record. Med Decis Making 2010;30(6):639-50

- Niemeijer M, Loog M, Abramoff MD, Viergever MA, Prokop M, van Ginneken B. On combining Computer-Aided Detection Systems. IEEE Trans Med Imaging 2010;30(2):215-23.

- van der Siis H, van Gelder T, Vulto A, Berg M, Aarts J. Understanding handling of drug safety alerts: a simulation study. Int J Med Inform 2010;79(5):361-9.

- Wright A, Chen ES, Maloney FL. An automated technique for identifying associations between medications, laboratory results and problems. J Biomed Inform 2010;43(6):891-901.

Appendix: Content Summaries of Selected Best Papers for the IMIA Yearbook 2011, Section Decision Support*

Elkin PL, Liebow M, Baver BA, Chaliki S, Wahner-Roedler D, BundrickJ, Lee M, Brown SH, Froehling D, Bailey K, Famiglietti K, Kim $\mathrm{R}$, Hoffer E, Feldman M, Barnett GO

The introduction of a diagnostic decision support system (DXplain ${ }^{T M}$ ) into the workflow of a teaching hospital service can decrease the cost of service for diagnostically challenging Diagnostic Related Groups (DRGs) Int J Med Inform 2010;79:772-7

This article demonstrates how the introduction of a diagnostic decision support system (DXplain) as a support for residents in a hospital environment can decrease the costs and the charges for a diagnostically challenging patient case. This study has been conducted in the general medicine service at Saint Mary's Hospital in Minnesota, USA.

According to the authors, residents usually do not have enough time to consider and investigate multiple different diagnoses.

* The complete papers can be accessed in the Yearbook's full electronic version, provided that permission has been granted by the copyright holder(s).
DXplain is a computer-based, medical education, reference and decision support system. In its function as a support tool, it uses an interactive format to collect clinical information and then uses data of about 5'000 clinical manifestations (history, examination findings, laboratory and imaging data) to generate a differential diagnosis ranked by probability of the diagnosis, which is based on the patients' data. As a medical textbook and reference, it provides a comprehensive description and selected references for more than 2'200 different diseases, emphasizing the signs and symptoms present in each disease, the etiology, the pathology and the prognosis. The aim of working with an expert system is for the resident to generate quicker and easier an adequate diagnosis.

The study compared the costs and charges between a period without DXplain access and a period with DXplain access. Using the support system, costs were reduced by $12 \%$ and charges by $10 \%$. The length of stay also decreased. There were no other changes, so the access to DXplain is the most likely explanation for the decrease. The average level of experience was identical in the intervention and the control period. Costs per case were on average almost $\$ 1000$ less in the intervention period than in the control period, which represents savings of more than $\$ 2^{\prime} 250^{\prime} 000$. In addition, residents and professionals can focus on other important issues like management of existing problems.

But one should take into account that the study only took place in one 
hospital and that no feedback was obtained from patients.

In conclusion, this study demonstrates that costs and charges decreased significantly for diagnostically challenging cases with DX-plain access. Such diagnostic decision support system can be used for educational purposes, the inpatient admissions can be managed more efficiently, and the system can be updated and developed constantly.

Niemeiier M, Loog M, Abramoff MD, Viergever MA, Prokop M, van Ginneken B On combining Computer-Aided Detection Systems

IEEE Trans Med Imaging 2010;30(2):215-23

The aim of this work is to investigate the added value of combining computeraided detection (CAD) systems for radiological diagnosis. CAD is increasingly used in clinical practice. In this study, two algorithms, ANODE09 and ROC09, are combined and used for the detection of pulmonary nodule. The authors envisioned four different scenarios when combining CAD systems.

For this method, 3 steps were followed: 1) transforming the degree of suspicion values for each CAD system. 2) merging findings from different systems, which probably refer to the same lesion, and 3) „Designing a fixed combination rule or supervised combiner that estimates a new degree of suspicion for the merged findings." Once all possible combinations from ANODE09 and ROC09 were investigated, a statistical analysis is performed to compare the best individual systems with the best combinations.

Combining CAD systems results in a significant increase in performance, compared to the best individual CAD systems. However, the authors recommend that in order to accomplish a high performance it is necessary that both CAD systems are considered as good systems. Otherwise the performance will tend to be in between (the good and the bad system).

The report concludes that a combination of systems outperforms individual approaches.

Wright A, Chen ES, Maloney FL An automated technique for identifying associations between medications, laboratory results and problems J Biomed Inform 2010;43(6):891-901

The patient problem list is an important component of clinical practice, as it facilitates decision support and quality measurement. Evidence suggests that patients with accurate and complete problem lists have better outcomes. However, the problem lists are often incomplete.

The objective of this paper is to determine whether association rule mining, a data mining technique, can be used to identify associations between medications, laboratory results, and problems. Such associations can be useful for identifying potential gaps in the problem list.

Association rule mining was performed on structured electronic health record data within a sample of 100'000 patients. These patients received care at the Brigham and Women's Hospital, in Boston, USA.

Candidate medication-problem and laboratory-problem associations were generated. High-scoring candidate pairs were compared to a gold standard: the Lexi-Comp drug reference database for medications and Mosby's Diagnostic and Laboratory Test Reference for Laboratory results.

Association rule mining identified a large number of clinically accurate associations, which leads to the conclusion that it is a useful methodology to identify clinical associations between medications, laboratory results and problems.

Matheny ME, Miller RA, Ikizler TA, Waitman LR, Denny JC, Schildcrout JS, Dittus RS, Peterson JF

Development of inpatient risk stratification models of acute kidney injury for use in electronic health record

Med Decis Making 2010;30(6):639-50

Acute kidney injury affects between 1\% and $5 \%$ of all hospitalized patients and $5 \%$ to $20 \%$ of patients requiring intensive care. Patients with hospital-acquired acute kidney injury have a higher risk of mortality and further medical complications.

The objective of this paper is to identify high-risk patients, prior to the development of an acute kidney injury, with a predictive tool that is implemented in an electronic health record (EHR).

A retrospective cohort of 61'179 patients was collected at the Vanderbilt University Medical Center during four years. Using administrative, computerized provider order entry and laboratory test data, the authors developed a 3-level risk stratification model to predict each of two severity levels of in-hospital acute kidney injury.
The models indentify patients with high risk of acute kidney injury, using only commonly available electronic data.

van der Sijs H, van Gelder T, Vulto A, Berg M, Aarts J

\section{Understanding handling of drug safety} alerts: a simulation study

Int J Med Inform 2010;79(5):361-9

The purpose of this report is to validate the correctness of drug safety alert handling and to study the error type in a computerized physicians order entry (CPOE) system in a simulated work environment. For this study, 18 physicians were selected (two-thirds came from internal medicine and the rest from surgery). 35 orders of predefined patient cases with 13 different drug safety alerts were entered in a CPOE in a noisy atmosphere and under time pressure in order to simulate real work conditions. Once the physicians entered an order in the system, they were interviewed about the drug safety alert generated, the reason and the cause of this and their knowledge of the alert. The reaction of the physicians was then classified into three groups.

1) "Override: the order is confirmed despite the generated alert.

2) Annulment: The order is adjusted resulting in the disappearance of the alert in the final order

3 ) Adjustment: the order is adjusted but does not result in the absence of the alert."

The expert defined the correctness of handling, reasoning, and the performance level using the SRK model (skill-based, rule-based, knowledge base behavior) of human performance in information processing. This model is often used in cognitive psychology.

As a result, 30\% of the alerts were handled inappropriately due to incorrect actions or incorrect reasoning. $63 \%$ of the errors were categorized as rule-based error, and $25 \%$ of the physicians did suffer from alert fatigue.

In conclusion this report shows that the reasons why alerts were handled incorrectly are that physicians ignored the alerts, misinterpreted the alerts (unclear alert text and text read incompletely) or the alert presentation was unclear. Physicians revealed that the study resembled to the normal work environment. The authors suggest to improve training and produce more concise alert texts. 